\title{
Predictors for Likelihood of Corneal Transplantation in Keratoconus
}

\author{
E. G. WOODWARD, L. C. MOODALEY, A. O'HAGAN \\ London and Coventry
}

\begin{abstract}
Summary
A group of 70 patients who had attended the Keratoconus Clinic at Moorfields Eye Hospital over a 10-year period were analysed retrospectively. At the end of this period 23 eyes had undergone corneal transplantation.

This gave an overall probability of $16 \%$, various information obtained at the initial visit was then analysed for a possible relationship with the probablity of needing a transplant within ten years.

The only significant relationship found was the initial corneal radius as determined by the back optic radius of the first contact lens. A graph was constructed plotting probability of corneal transplant against each radius at the first visit, which could assist clinicians in advising patients.
\end{abstract}

Although there have been many papers giving the incidence of corneal transplantation in groups of keratoconus patients ${ }^{1,2}$ as far as we are aware no attempt has been made to predict the likelihood of subsequent penetrating keratoplasty at the initial examination. To investigate this specific risk ratio, a group of patients who had at least a ten-year follow-up in the Keratoconus Clinic at the Department of Contact Lens and Prosthetics, Moorfields Eye Hospital were evaluated. This group of patients had earlier been reported on by Cox. ${ }^{3}$ Cox had reported on 88 patients and at 10 years 70 of these patients were still attending the Clinic. Of these patients 16 (23 eyes) had undergone corneal transplantation. During this period the criteria for transplantation were in general a visual acuity of $6 / 18$ or worse, or intolerance to contact lens correc- tion. No patients were grafted as part of the management of acute hydrops in this series.

\section{Materials and methods}

The patients investigated first presented at Moorfields Eye Hospital between 1973 and 1978. From their notes it was possible to extract:

(a) Age at presentation

(b) Mode of correction at first visit, and at 2,5 and 10 years

(c) Snellen visual acuity at first visit, 2, 5 and 10 years

(d) Back optic radius of initial contact lens fitted and subsequent changes and chronology

(e) Timing of events such as penetrating keratoplasty and acute hydrops

It was decided to use back optic radius of

From: Department of Optometry and Visual Science, City University, Dame Alice Owen Building, 311-321 Goswell Road, London EC1V 7DD (EGW), Contact Lens Department, Moorfields Eye Hospital, Cayton Street, London EC1V 2PD (LCM) and Department of Statistics, University of Warwick, Coventry CV4 7AL (AO'H).

Correspondence to: Professor EG Woodward, Department of Optometry and Visual Science, City University, Dame Alice Owen Building, 311-321 Goswell Street, London EC1V 7DD. 
the lens fitted as an indicator of the progression of the condition, rather than keratometry. Keratometry becomes increasingly inaccurate as keratoconus progresses. The optical theory is based on catoptric images reflected from an axially symmetrical system, a situation which does not pertain, even in moderate keratoconus. The fitting philosophy used in the clinic was agreed by all clinic staff so it was considered that changes in back optic radius did represent proportionate changes in corneal contour. Furthermore patients attended the clinic wearing their contact lenses so this would also render keratometric values obtained immediately after lens removal unrepresentative.

The data were then analysed by one of the authors (AO'H) using the method of logistic regression to study possible predictors of eventual need for keratoplasty within 10 years. (For a full explanation of the statistical method see the technical appendix).

\section{Results}

Mean age at presentation was 21.2 years (SD 5.08).

Over the period studied there was no evidence that, for the same patient, the natural history of the disease was not independent between the two eyes. As a result of this, eyes were considered separately.

Where the patient was wearing corneal lenses the back optic radius was recorded at each visit. It was found that changes in back optic radius seemed to occur randomly with time and the amount of change also appeared random.

Of the 23 transplants 21 occurred within six years of diagnosis and two occurred at the 10 th year. It is possible, of course, that the need for a graft could arise after the 10 -year period but perusal of the data suggests that most eyes stabilise after that time.

Eyes were then divided into groups by initial correction.

Table I Mode of correction (eyes)

\begin{tabular}{lrrrr}
\hline & Initial & 2 yrs & 5 yrs & 10 yrs \\
\hline None & 17 & 15 & 12 & 11 \\
Spectacles & 18 & 13 & 20 & 18 \\
Corneal lens & 96 & 109 & 105 & 110 \\
Scleral lens & 7 & 5 & 3 & 1 \\
Hydrogel lens & 2 & 2 & 0 & 0 \\
\hline
\end{tabular}

I - No correction of spectacles

II - Corneal contact lenses

III - Other forms of contact lenses (scleral or hydrogel)

One eye was not included in this calculation as visual acuity at presentation was count fingers and the patient was listed for transplantation at the first visit.

In Group I-Probability of transplant within $10 \mathrm{yrs}=0.06=6 \%$.

In Group III-Probability of transplant within 10 yrs $=0.50=50 \%$ (a rounded figure due to low numbers in this group).

Group II was submitted to further analysis. Although there was an overall probability of $16 \%$, various predictors at the time of the first visit to the clinic were analysed for a possible relationship with the probability of needing a transplant within ten years. Age, sex, the back central optic radius (BCOR) of the initially fitted lens, and initial visual acuity were investigated, but only the initial B COR had a significant effect. A keratoconic eye with a corneal radius within normal limits, at first visit, has the same risk of having a transplant as Group I, i.e. 6\%. Whereas if the initial B CO R is $6 \mathrm{~mm}$ or less the risk is comparable to an eye in Group III, i.e. $50 \%$.

These findings are illustrated graphically in Fig. 1, which plots estimated probability of corneal transplant against B C O R at initial visit.

\section{Discussion}

Patients often have the impression or may even have been told that they will inevitably need surgery for this condition. Although this will vary with the treatment protocol of an individual unit the results of this study give probabilities which are statistically significant when related to measurements taken at the first visit. In terms of patient management this enables the clinician to inform the patient of the likelihood of requiring a corneal transplant within 10 years of diagnosis. It has been

Table II Eyes transplanted by group

\begin{tabular}{rcc} 
& Eyes & Transplanted \\
\hline I & 34 & 2 \\
II & 96 & 15 \\
III & 9 & 5 \\
\hline
\end{tabular}




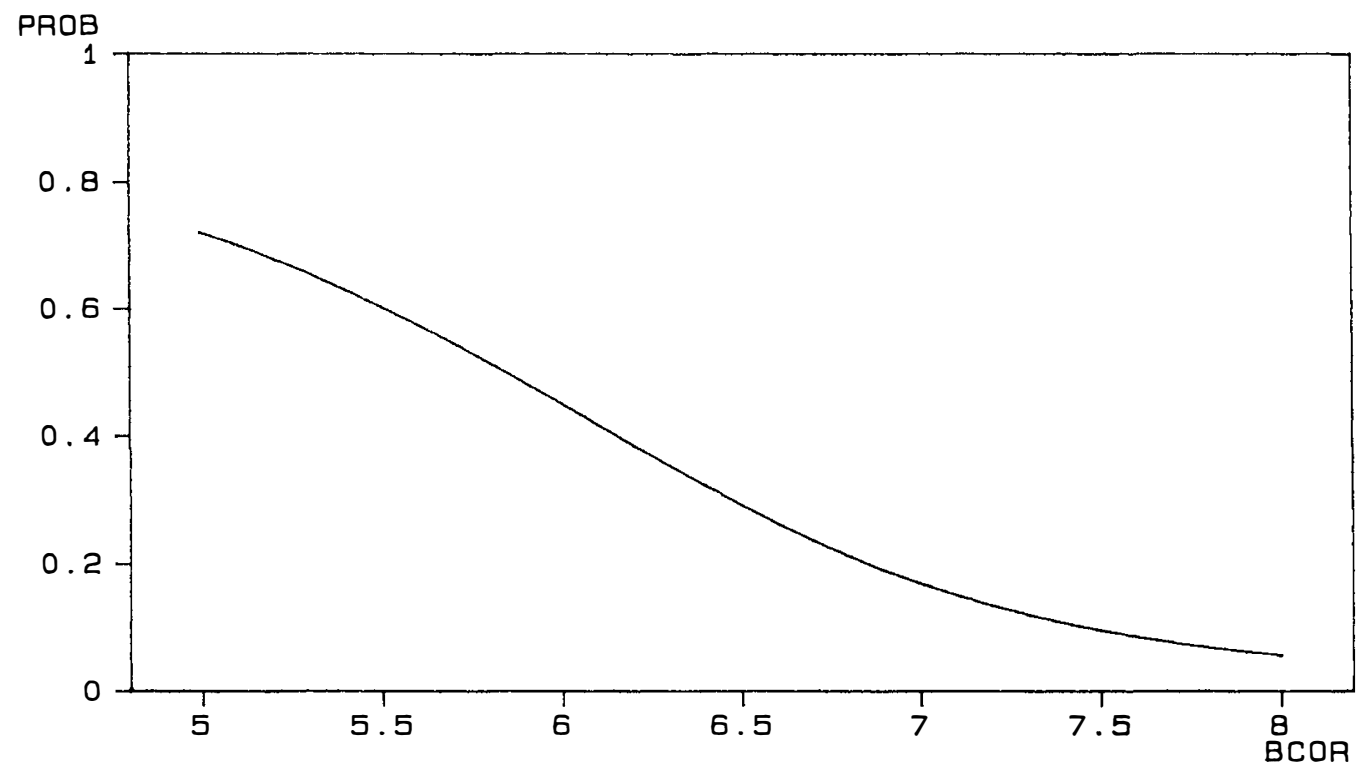

Fig. 1 Estimated probability of transplant for given $B C O R$.

shown that many keratoconus patients are young and economically active and this information should be of help to them in planning their life and career for the next 10 years.

\section{Appendix}

To study possible predictors of eventual need for grafts, we used the method of logistic regression, which is a common technique for problems with binary responses. In the problem at hand, the binary response was whether or not a given eye needed a graft within ten years. Attention was restricted to eyes for which the initial treatment was to fit a hard (gas-permeable) contact lens. The data thus comprised 96 cases, being that subset of the 140 eyes from 70 patients in the study which were initially fitted with hard lenses.

The method allowed us to relate the probability of a graft to one or more predictor variables. The first stage analysis was therefore to identify significant predictors, and for this exploratory work the standard computer package GLIM was used. It was found that sex and age at diagnosis did not have any significant effects, and nor did visual acuity using the initially fitted lens. The one significant predictor variable was the corneal radius (B COR) of the initially fitted lens. The square of B C O R was also introduced as a predictor variable, but was found to have no significant additional effect on the response. The final model was the very simple one

Prob (graft for case $i)=\exp \left(z_{i}\right) /\left\{1+\exp \left(z_{i}\right)\right\}$, (1) where

$$
z_{i}=\alpha+\beta x_{i}
$$

and where $x_{i}$ is the B COR for the $i$-th eye.

The classical statistical methods implemented in GLIM yield asymptotically unbiased estimates and asymptotic confidence intervals for $\alpha$ and $\beta$. We adopted instead a Bayesian approach, which allows exact and more detailed inferences to be derived. The details of this methodology are presented in another, more technical paper elsewhere. ${ }^{4}$ The estimates for $\alpha$ and $\beta$ were $E(\alpha)=8.23, \quad E(\beta)=-1.41$. The classical asymptotic test provided through GLIM showed that $\beta$ was significantly different from zero $(P=0.008)$. The Bayesian analogue is a one-sided result: the probability that $\beta \geqslant 0$ was found to be 0.004 .

Inserting the estimates of $\alpha$ and $\beta$ into (2) gives the estimate of $z_{i}$ for a patient with initial B COR $x_{i}$. The result may then be inserted into (1) to estimate the probability of a graft for that patient. This procedure provides a good approximation to a slightly more accurate estimate given in the more technical paper. Figure 1 presents the result graphically, enabling the probability of a graft within 
ten years for a patient with given initial B C O R to be read off directly.

$95 \%$ probability bounds on this estimate were computed for various initial B C O R. Bounds at three different values are given in the table below.

Table III Estimates and 95\% bounds on probability of keratoplasty

\begin{tabular}{cccc}
$\begin{array}{c}\text { Initial } \\
B C O R\end{array}$ & $\begin{array}{c}\text { Lower } \\
\text { Bound }\end{array}$ & Estimate & $\begin{array}{c}\text { Upper } \\
\text { Bound }\end{array}$ \\
\hline $5.5 \mathrm{~mm}$ & 0.23 & 0.60 & 0.90 \\
$6.5 \mathrm{~mm}$ & 0.15 & 0.29 & 0.46 \\
$7.5 \mathrm{~mm}$ & 0.04 & 0.10 & 0.18 \\
\hline
\end{tabular}

The width of these bounds reflects the limited amount of data available. In particular, the few patients initially presenting with B CO R values lower than about $6.5 \mathrm{~mm}$ has resulted in high uncertainty about graft probabilities at those values.

It is important to recognise the dependence of these results on the treatment protocol of an eye unit. Obviously, a protocol which was more reluctant to use grafts, or delayed their use, would produce different results. Any improvement in treatment would have a similar effect.

\section{References}

${ }^{1}$ Kennedy RH, Bourne WM, Dyer JA: A 48-year clinical and epidemiological study of keratoconus. Am J Ophthalmol 1986, 101: 267-73.

${ }^{2}$ Smiddy WE, Harbury TR, Kracker GP, Stark WJ: Keratonconus: contact lens or keratoplasty. Ophthalmology 1988, 95: 487-92.

${ }^{3}$ Cox SN: Management of keratoconus. J Br Contact Lens Assoc 1984, 7: 56-64.

${ }^{4}$ O'Hagan A, Woodward EG, Moodaley LC: Practical Bayesian analysis of a simple logistic regressionpredicting corneal transplants. Warwick Statistics Research Report 163, Department of Statistics, University of Warwick 1989. (Submitted to Statistics in Medicine.) 